\title{
Reduced Order Modeling of a Shipboard Power System
}

\author{
S. Abdelwahed, A. Asrari, J. Crider, R.A. Dougal, M.O. Faruque, Y. Fu, J. Langston, Y.Lee, H.A. Mohammadpour, \\ A. Ouroua, E. Santi, K. Shoder, S.D. Sudhoff, Y. Zhang, H. Zheng, E. Zivi \\ Electric Ship Research and Development Consortium \\ www.esrdc.com
}

\begin{abstract}
Reduced-order average-value models form the basis of a computationally efficient approach for studying shipboard power systems. As a result of neglecting fast states, this approach generally involves solving a set of differential algebraic equations. Although the reduced-order average-value modeling approach has been well studied in the literature, as simulation languages evolve the most programmatic approach to addressing the non-linear algebraic portion of the model changes as well. In this work, a number of approaches for implementing reduced-order average-value models of a small power system are compared.
\end{abstract}

\section{INTRODUCTION}

Modeling of shipboard power systems is integral to the ship design process. However, depending on objectives to be achieved, the required fidelity of the model for a given application varies largely. For example, waveform level power converter models represent the dynamics of the system on a component switching level and yield results comparable to the actual measurements. Therefore, this level of fidelity is suitable for analyzing the detailed transient response of the system. Examples of such waveform level simulations in shipboard power systems are given in [1] and [2]. These high fidelity models are complex and computationally expensive. These drawbacks make them inefficient for applications requiring quick evaluation as in the ship system assessment in the early design stage. In order to develop computationally efficient shipboard power system models, a reduced-order modeling approach is used wherein the fast dynamics of the system are neglected and the semiconductor switching is represented on an average-value basis. This modeling technique is often used in conjunction with reference frame theory so that state variables are constant in steady-state. As the result of these simplifications, it is possible to reduce numerical complexity and utilize variable step algorithms, thus obtaining a representation of the system dynamics without the waveform level model computation costs.

The use of reduced-order average-value models has been well established in the literature. However, there are important aspects of these models which are a function of simulation platform. In particular, this class of models typically involves the solution of non-linear algebraic equations as part of the time-domain integration. While there are a number of techniques to do this, the best approach to

This work was sponsored by the Office of Naval Research through Grant N00014-08-1-0080 doing so is a function of platform and changes as simulation platforms evolve. In pursuit of more efficient and accurate approaches to reduced-order average-value models, this work compares a number of methods in the context of a small shipboard power system.

The organization of this work is as follows. First, in Section II, a sample system is described. In Section III, a reduced-order average-value model of the system is set forth. A variety of model implementation strategies are described in Section IV. The system simulation scenario and the time responses of the simulation results are included in Section V. In Section VI, these strategies are numerically compared using a defined error metric. Section VII concludes the work.

\section{SYSTEM DESCRIPTION}

Fig. 1 depicts the simple power system used in this work to compare different reduced order model implementations. This system was chosen to be simple enough to facilitate a straightforward system description but involved enough to be interesting. It consists of a synchronous machine (SM) being operated as a generator, connected to three loads by a cable (CBL). The three loads are an induction machine (IM), an RL load (RL), and a transformer rectifier load (TR). A complication that arises in this system modeling is the need to solve the set of non-linear system interconnection algebraic equations at each time step. There are several different ways to approach this problem, and this is one factor which leads to multiple reduced-order model approaches as described in this work.

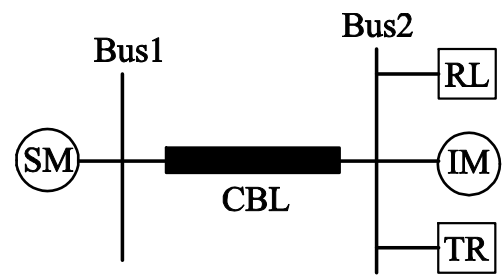

Figure 1. Notional Power System One-line Diagram

The synchronous machine and exciter component parameters are listed in Table 1 followed by the parameters for the rest of the system components in Table 2. The definitions of the model parameters are given in the model description in the subsequent section. 
Table 1. Synchronous Machine Component Parameters

\begin{tabular}{|c|c|c|c|}
\hline \multicolumn{2}{|c|}{ SM } & \multicolumn{2}{c|}{ Exciter } \\
\hline$P_{S M}(V A)$ & $25 e^{6}$ & $K_{P R}(p u)$ & 200 \\
\hline$V_{L L}(V)$ & 4160 & $K_{I R}(p u)$ & 0.8 \\
\hline$\omega_{m b}(\mathrm{rad} / \mathrm{s})$ & $60 \pi$ & $K_{D R}(p u)$ & 0.001 \\
\hline$P$ & 4 & $T_{D R}(s)$ & 0.001 \\
\hline$p f$ & 0.8 & $K_{A}(p u)$ & 1 \\
\hline$r_{s}(m \Omega)$ & 3 & $T_{A}(s)$ & 0.0001 \\
\hline$L_{l s}(m H)$ & 0.2 & $V_{r m x}(p u)$ & 5 \\
\hline$L_{m q}(m H)$ & 2 & $V_{r m n}(p u)$ & 0 \\
\hline$L_{m d}(m H)$ & 2 & $T_{E}(s)$ & 1 \\
\hline$r_{k q}(m \Omega)$ & 5 & $K_{E}(p u)$ & 1 \\
\hline$L_{l k q}(m H)$ & 0.04 & $S E_{A}(p u)$ & 1.0119 \\
\hline$r_{k d}(m \Omega)$ & 5 & $S E_{B}(p u)$ & 0.0875 \\
\hline$L_{l k d}(m H)$ & 0.04 & SM Mechanical \\
\hline$r_{f d}(m \Omega)$ & 20 & $K_{P}(\mathrm{Nm} \cdot S / \mathrm{rad})$ & $10^{4}$ \\
\hline$L_{l f d}(m H)$ & 0.15 & $K_{I}(\mathrm{Nm} / \mathrm{rad})$ & $10^{4}$ \\
\hline$v_{f d B}(\mathrm{~V})$ & 90.1 & \multicolumn{3}{|c|}{$J\left(\mathrm{~kg} \cdot \mathrm{m}^{2}\right)$} & 4221.7 \\
\hline
\end{tabular}

Table 2. System Component Parameters

\begin{tabular}{|c|c|c|c|}
\hline \multicolumn{2}{|c|}{ CBL } & \multicolumn{2}{|c|}{ IM } \\
\hline$R(m \Omega)$ & 0.865 & $P(M W)$ & 5 \\
\hline$L(\mu H)$ & 2.3 & $\omega_{r m b}(\mathrm{rad} / \mathrm{s})$ & $60 \pi$ \\
\hline \multicolumn{2}{|c|}{ RL } & $\mathrm{P}$ & 4 \\
\hline$P(M W)$ & 5 & $T_{b}(k N m)$ & 26.53 \\
\hline$R(\Omega)$ & 2.8 & $T_{L}(\mathrm{Nm})$ & $T_{b}\left(\omega_{r m} / \omega_{r m b}\right)^{3}$ \\
\hline$L(m H)$ & 3.6 & $R(m \Omega)$ & 31.8 \\
\hline$p f$ & 0.9 & $L_{l s}(m H)$ & 0.658 \\
\hline \multicolumn{2}{|c|}{ TR } & $L_{M}(m H)$ & 38 \\
\hline$P(M W)$ & 10 & $r_{r}^{\prime}(m \Omega)$ & 24.1 \\
\hline$v_{d c}(k V)$ & 5.62 & $L_{l r}^{\prime}(m H)$ & 0.658 \\
\hline$i_{d c}(k A)$ & 1.78 & $J\left(\mathrm{~kg} \cdot \mathrm{m}^{2}\right)$ & 250 \\
\hline$R(\Omega)$ & 3.16 & & \\
\hline$L_{c}(\mu H)$ & 76.5 & & \\
\hline$L_{d c}(m H)$ & 0.383 & & \\
\hline$C(m F)$ & 0.384 & & \\
\hline
\end{tabular}

\section{REDUCED-ORDER MODEL DESCRIPTION}

Creating reduced-order models involves neglecting fast system dynamics, which is accomplished by neglecting the appropriate time-derivative terms. In order to remove the time-derivative terms without affecting steady-state behavior, it is important that the system model be formulated so that the state-variables are constant in the steady state. This normally requires expressing the system equations in terms of a synchronous reference frame, so that ac quantities are represented by dc quantities. It also requires representing the switching of power converters on an average value basis. The average value operator is defined as

$$
\hat{x}(t)=\int_{t-T}^{t} x(\tau) d \tau
$$

where $T$ is the switching period of the component of interest. The reduced-order average models of the sample system components will now be set forth.

\section{A. Synchronous Machine}

The derivation of both detailed and reduced-order models of a synchronous machine can be found in [3]. These models are based in the rotor reference frame. It is convenient to represent the reduced-order synchronous machine stator voltage equations in voltage-behind-reactance form utilizing the subtransient quantities. For a synchronous machine with one damper widing in each axis, the subtransient inductances and flux linkages are expressed

$$
\begin{gathered}
L_{q}^{\prime \prime}=L_{l s}+\frac{L_{m q} L_{l k q}^{\prime}}{L_{l k q}^{\prime}+L_{m q}} \\
L_{d}^{\prime \prime}=L_{l s}+\frac{L_{m d} L_{l f d}^{\prime} L_{l k d}^{\prime}}{L_{m d} L_{l f d}^{\prime}+L_{m d} L_{l k d}^{\prime}+L_{l f d}^{\prime} L_{l k d}^{\prime}} \\
\lambda_{q}^{\prime \prime}=\frac{L_{m q}}{L_{m q}+L_{l k q}^{\prime}} \lambda_{k q}^{r r} \\
\lambda_{d}^{\prime \prime}=\frac{L_{m d}\left(\frac{\lambda_{k d}^{\prime r}}{L_{l k d}^{\prime}}+\frac{\lambda_{f d}^{\prime r}}{L_{l f d}^{\prime}}\right)}{1+\frac{L_{m d}}{L_{l f d}^{\prime}}+\frac{L_{m d}^{\prime}}{L_{l k d}^{\prime}}}
\end{gathered}
$$

The stator voltage equations, flux linkages, and the derivative of flux linkages can then be written as

$$
\begin{gathered}
\boldsymbol{V}_{q d s}^{r}=\left[\begin{array}{cc}
r_{s} & \omega_{r} L_{d}^{\prime \prime} \\
-\omega_{r} L_{q}^{\prime \prime} & r_{s}
\end{array}\right] \boldsymbol{i}_{q d s}^{r}+\left[\begin{array}{c}
\omega_{r} \lambda_{d}^{\prime \prime} \\
-\omega_{r} \lambda_{q}^{\prime \prime}
\end{array}\right] \\
\lambda_{q s}^{r}=L_{q}^{\prime \prime} i_{q s}^{r}+\lambda_{q}^{\prime \prime} \\
\lambda_{d s}^{r}=L_{d}^{\prime \prime} i_{d s}^{r}+\lambda_{d}^{\prime \prime} \\
\frac{d \lambda_{k q}^{\prime r}}{d t}=\frac{-r_{k q}^{\prime}}{L_{l k q}^{\prime}}\left(\lambda_{k q}^{\prime r}-L_{q}^{\prime \prime} i_{q s}^{r}-\lambda_{q}^{\prime \prime}+L_{l s} i_{q s}^{r}\right) \\
\frac{d \lambda_{k d}^{\prime r}}{d t}=\frac{-r_{k d}^{\prime}}{L_{l k d}^{\prime}}\left(\lambda_{k d}^{\prime r}-L_{d}^{\prime \prime} i_{d s}^{r}+\lambda_{d}^{\prime \prime}-L_{l s} i_{d s}^{r}\right)
\end{gathered}
$$




$$
\begin{gathered}
\frac{d \lambda_{f d}^{\prime r}}{d t}=v_{f d}^{\prime r}-\frac{r_{f d}^{\prime}}{L_{l f d}^{\prime}}\left(\lambda_{f d}^{\prime r}-L_{d}^{\prime \prime} i_{d s}^{r}+\lambda_{d}^{\prime \prime}-L_{l s} i_{d s}^{r}\right) \\
T_{e}=\frac{3}{2} \frac{P}{2}\left(\lambda_{d s}^{r} i_{q s}^{r}-\lambda_{q s}^{r} i_{d s}^{r}\right)
\end{gathered}
$$

Therefore, the synchronous machine model inputs are the $q$ and $d$ axis load currents defined as the sum of all the load currents (CBL, IM, RL, and TR). The states of the synchronous machine model are the three rotor flux linkages defined in equations (9) through (11).

The mechanical dynamics of the synchronous machine, as found in [3], are expressed as

$$
\frac{d \omega_{r m}}{d t}=\frac{T_{e}-T_{m}}{J}
$$

where $J$ is the inertia of the machine in $\mathrm{kg} \cdot \mathrm{m}^{2}$ and $T_{m}$ is the prime mover torque of the machine in $\mathrm{Nm}$, obtained from the proportional and integral gain of the difference between the mechanical speed and the commanded speed as shown in Fig. 2.

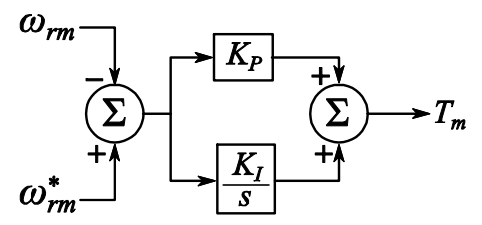

Figure 2. Synchronous Machine Mechanical Model

It is relevant to identify that the presence of a single generator in the power system enables the synchronous reference frame angle of the system to be the rotor angle of the generator.

\section{B. Exciter}

The synchronous machine also contains an exciter model. This exciter model is depicted in Fig. 3 and is closely based upon IEEEAC8B [4].

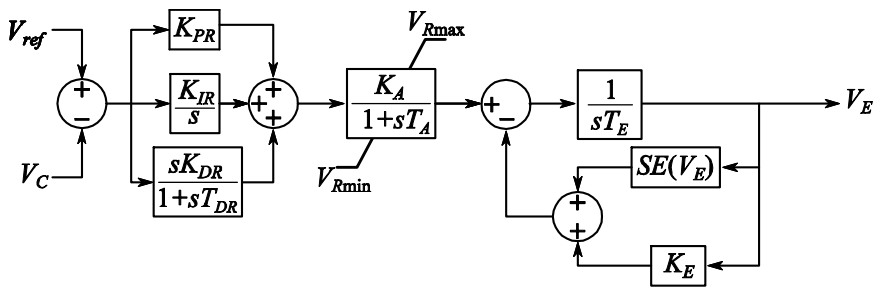

Figure 3. Synchronous Machine Exciter Model

The reference voltage $V_{\text {ref }}$ and measured voltage $V_{C}$ in the above figure are per-unit line-to-line voltages. The physical field voltage is found by scaling the per-unit value of $V_{E}$ by the base field voltage $v_{f d B}^{r^{\prime}}$ found by

$$
v_{f d B}^{r^{\prime}}=\frac{v_{\text {rated }} r_{f d}^{\prime}}{\omega_{r} L_{m d}}
$$

\section{System Interconnection Cable}

The cable connecting the source (SM) to the loads (RL, $\mathrm{IM}$, and TR) is represented as an RL line and is depicted in Fig. 4. The reduced-order model may be expressed in the synchronous reference frame as

$$
\begin{gathered}
v_{q 1}^{e}-v_{q 2}^{e}=r i_{q 12}^{e}+\omega_{e} L i_{d 12}^{e} \\
v_{d 1}^{e}-v_{d 2}^{e}=r i_{d 12}^{e}-\omega_{e} L i_{q 12}^{e}
\end{gathered}
$$

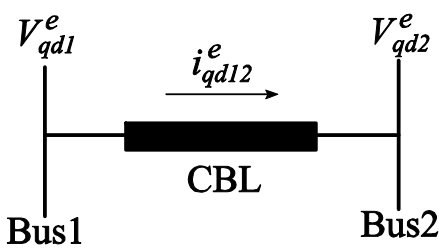

Figure 4. System Interconnection Cable

The inputs to the cable model are the $q$ and $d$ axis voltages at Bus 1 and the $q$ and $d$ axis currents into the synchronous machine. The outputs of the cable model are the $q$ and $d$ axis voltages at Bus 2.

\section{RL Load}

The reduced-order RL load model equations are defined as

$$
\begin{gathered}
v_{q}^{e}=r i_{q}^{e}+\omega_{e} L i_{d}^{e} \\
v_{d}^{e}=r i_{d}^{e}-\omega_{e} L i_{q}^{e}
\end{gathered}
$$

The RL load model inputs are the $q$ and $d$ axis voltages at Bus 2 and the model outputs are the $q$ and $d$ axis currents into the load.

\section{E. Induction Machine}

The reduced-order model of an induction machine is derived in [3] is expressed in the following equations.

$$
\begin{gathered}
\boldsymbol{V}_{q d s}^{e}=r_{s} i_{q d s}^{e}+\omega_{e} \lambda_{d q s}^{e} \\
\lambda_{d q s}^{e}{ }^{e}=\left[\begin{array}{ll}
\lambda_{d s}^{e} & -\lambda_{q s}^{e}
\end{array}\right] \\
\boldsymbol{V}_{q d r}^{e^{\prime}}=r_{r}^{\prime} \boldsymbol{i}_{q d r}^{e^{\prime}}+\left(\omega_{e}-\omega_{r}\right) \lambda_{d q r}^{e^{\prime}} \\
\lambda_{d q r}^{e^{\prime} T}=\left[\begin{array}{ll}
\lambda_{d r}^{e^{\prime}} & -\lambda_{q r}^{e^{\prime}}
\end{array}\right] \\
i_{q d r}^{e^{e^{\prime}}}=\frac{1}{L_{l r}^{\prime}+L_{M}} \lambda_{q d r}^{e^{\prime}}-\frac{L_{M}}{L_{l r}^{\prime}+L_{M}} i_{q d s}^{e}
\end{gathered}
$$




$$
\begin{aligned}
\frac{d \lambda_{q r}^{e^{\prime}}}{d t} & =-r_{r}^{\prime} i_{q r}^{e^{\prime}}-\left(\omega_{e}-\omega_{r}\right) \lambda_{d r}^{e^{\prime}} \\
\frac{d \lambda_{d r}^{e^{\prime}}}{d t} & =-r_{r}^{\prime} i_{d r}^{e^{\prime}}+\left(\omega_{e}-\omega_{r}\right) \lambda_{q r}^{e^{\prime}} \\
T_{e} & =\frac{3}{2} \frac{P}{2}\left(\lambda_{d s}^{e} i_{q s}^{e}-\lambda_{q s}^{e} i_{d s}^{e}\right)
\end{aligned}
$$

with $q$ and $d$ axis vector quantities in bold.

The mechanical dynamics of the induction machine, as defined in [3], are

$$
\frac{d \omega_{r}}{d t}=\frac{P}{2} \frac{T_{e}-T_{m}}{J}
$$

where $J$ is the inertia of the machine in $\mathrm{kg} \cdot \mathrm{m}^{2}$ and $T_{m}$ is the load torque of the induction machine defined as a speed cubed load.

The induction machine model inputs are the $q$ and $d$ axis voltages at Bus 2 and the outputs of the model are the currents into the load. The states in the induction machine model are the rotor flux linkages $(24,25)$ and the derivative of the speed of the machine (27).

\section{F. Transformer Rectifier}

The transformer rectifier load model is depicted in Fig. 5. The model equations for the transformer rectifier in the synchronous reference frame are defined in [3] and represented by equations (28) through (41).

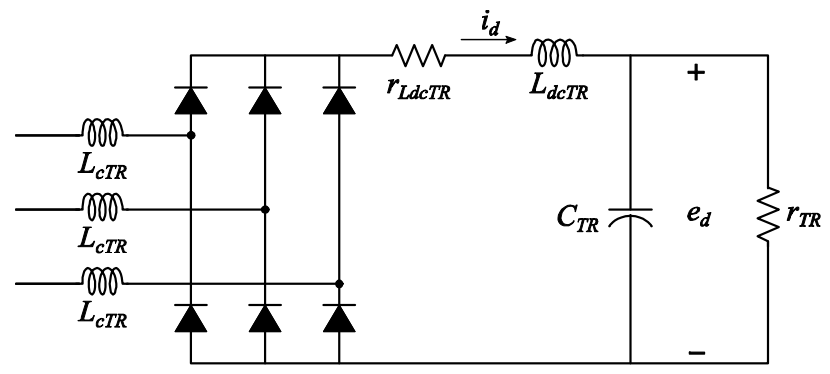

Figure 5. Transformer Rectifier Load

The transformer model begins by defining $E \angle \phi_{g}$ as the rms line-to-neutral ac input voltage phasor. The magnitude and phase can be written as

$$
\begin{gathered}
E=\frac{1}{\sqrt{2} N_{p s}} \sqrt{\left(v_{q g}^{e}\right)^{2}+\left(v_{d g}^{e}\right)^{2}} \\
\phi_{g}=\operatorname{ang}\left(v_{q g}^{e}+j v_{d g}^{e}\right)
\end{gathered}
$$

where $N_{p s}$ is the primary to secondary turns ratio in the event that the rectifier is connected to the bus via a transformer, in which case $L_{\text {CTR }}$ includes the total transformer leakage inductance viewed from the secondary side. The commutation and firing angles of the transformer rectifier are computed as

$$
\begin{gathered}
u=-\alpha^{*}+\operatorname{acos}\left(\cos \left(\alpha^{*}\right)-\frac{2 L_{c T R} \omega_{g} i_{d}}{\sqrt{6} E}\right) \\
\alpha=\alpha^{*}
\end{gathered}
$$

where $\alpha^{*}$ is the desired firing angle, $L_{c T R}$ is the commutating inductance, and $\omega_{g}$ is the radian frequency. If $\mu \geq \pi / 3$ or $\mu+\alpha \geq \pi$ then

$$
\begin{gathered}
u=\pi / 3 \\
\alpha=\frac{\pi}{3}-\operatorname{acos}\left(\frac{2 L_{c T R} \omega_{g} i_{d}}{\sqrt{6} E}\right)
\end{gathered}
$$

The dc link dynamics are represented by the following

$$
\begin{gathered}
\frac{d i_{d}}{d t}=\frac{\frac{3 \sqrt{6}}{\pi} E \cos \alpha-\left(r_{L d c T R}+\frac{3}{\pi} L_{c T R} \omega_{g}\right) i_{d}-e_{d}}{L_{d c T R}+2 L_{c T R}} \\
\frac{d e_{d}}{d t}=\frac{i_{d}-\frac{e_{d}}{r_{T R}}}{C_{T R}}
\end{gathered}
$$

where $r_{\text {LdcTR }}$ is the series resistance of the de link inductor $L_{d c T R}$ and $r_{T R}$ is the load resistance of the transformer rectifier load. The ac currents of the transformer rectifier in the generator reference frame are defined as in the following expressions.

$$
\begin{aligned}
\bar{i}_{q g, c o m}^{g} & =\frac{2 \sqrt{3}}{\pi} \bar{i}_{d}\left[\sin \left(u+\alpha-\frac{5 \pi}{6}\right)-\sin \left(\alpha-\frac{5 \pi}{6}\right)\right] \\
& +\frac{3}{\pi} \frac{\sqrt{2} E}{L_{c T R} \omega_{g}} \cos \alpha[\cos (u+\alpha)-\cos \alpha] \\
& +\frac{1}{4} \frac{3}{\pi} \frac{\sqrt{2} E}{L_{c T R} \omega_{g}}[\cos (2 \alpha)-\cos (2 \alpha+2 u)] \\
\bar{i}_{d g, c o m}^{g} & =\frac{2 \sqrt{3}}{\pi}{\overline{i_{d}}}_{[}\left[\cos \left(\alpha-\frac{5 \pi}{6}\right)-\cos \left(u+\alpha-\frac{5 \pi}{6}\right)\right] \\
& +\frac{3}{\pi} \frac{\sqrt{2} E}{L_{c T R} \omega_{g}} \cos \alpha[\sin (u+\alpha)-\sin \alpha] \\
& +\frac{1}{4} \frac{3}{\pi} \frac{\sqrt{2} E}{L_{c T R} \omega_{g}}[\sin (2 \alpha)-\sin (2 \alpha+2 u)] \\
& -\frac{3}{\pi} \frac{\sqrt{2} E}{L_{c T R} \omega_{g}} u
\end{aligned}
$$




$$
\begin{gathered}
\bar{i}_{q g, \text { cond }}^{g}=\frac{2 \sqrt{3}}{\pi} \bar{i}_{d}\left[\sin \left(\alpha+\frac{7 \pi}{6}\right)-\sin \left(u+\alpha+\frac{5 \pi}{6}\right)\right] \\
\bar{i}_{d g, \text { cond }}^{g}=\frac{2 \sqrt{3}}{\pi} \bar{i}_{d}\left[-\cos \left(\alpha+\frac{7 \pi}{6}\right)+\cos \left(u+\alpha+\frac{5 \pi}{6}\right)\right] \\
\bar{i}_{q g}^{g}=\bar{i}_{q g, \text { com }}^{g}+\bar{i}_{q g, \text { cond }}^{g} \\
\bar{i}_{d g}^{g}=\bar{i}_{d g, \text { com }}^{g}+\bar{i}_{d g, \text { cond }}^{g}
\end{gathered}
$$

The $q$ and $d$ axis currents are transformed back to the synchronous reference frame using the following

$$
{ }^{g} K^{a}=\left[\begin{array}{rr}
\cos \phi & -\sin \phi \\
\sin \phi & \cos \phi
\end{array}\right]
$$

where $\phi$ is the angle of the $q$ and $d$ axis voltages at Bus 2 that are the inputs to the TR model.

\section{IMPLEMENATATION STRATEGIES}

Six modeling strategies were pursued in this work. Table 3 lists the software platform, loop solver method, integration algorithm, and the signal with the most error for each reducedorder model implementation strategy. In the table, 'Package Solver' refers to a numerical solution of the non-linear algebraic equations based on the built-in routines of the respective software package. The term 'Dedicated Solver' refers to a numerical solution of the non-linear algebraic equations using a user defined routine. The term 'Delay' implies that a time delay is used to break an algebraic loop. The integration algorithm is listed in the fourth column. For those entries listed as 'Discrete', the system is represented as a discrete-time equivalent based on a forward Euler integration algorithm. In all other cases, the integration algorithm is formulated in terms of the state time derivative vector in accordance with the algorithm listed. In particular, in Table 3,

\begin{tabular}{|c|c|c|c|c|}
\hline $\begin{array}{l}\text { Method } \\
\text { Reference }\end{array}$ & $\begin{array}{l}\text { Software } \\
\text { Platform }\end{array}$ & $\begin{array}{c}\text { Algebraic } \\
\text { Loop Solver }\end{array}$ & $\begin{array}{c}\text { Integration } \\
\text { Algorithm }\end{array}$ & $\begin{array}{l}\text { Signal with } \\
\text { max error }\end{array}$ \\
\hline Method 1 & $\begin{array}{l}\text { MATLAB } \\
\text { Simscape }\end{array}$ & $\begin{array}{l}\text { Package } \\
\text { Solver }\end{array}$ & ode $15 \mathrm{~s}$ & $\begin{array}{l}\text { IM } q \text { axis } \\
\text { current }\end{array}$ \\
\hline Method 2 & $\begin{array}{l}\text { MATLAB } \\
\text { Simulink }\end{array}$ & $\begin{array}{l}\text { Dedicated } \\
\text { Solver }\end{array}$ & ode23tb & $\begin{array}{l}\text { TR } q \text { axis } \\
\text { currents }\end{array}$ \\
\hline Method 3 & $\begin{array}{l}\text { MATLAB } \\
\text { Simulink }\end{array}$ & Delay & Discrete & IM torque \\
\hline Method 4 & $\begin{array}{l}\text { MATLAB } \\
\text { Simulink }\end{array}$ & Delay & Discrete & IM torque \\
\hline Method 5 & $\begin{array}{l}\text { MATLAB } \\
\text { Simulink }\end{array}$ & Delay & ode $23 t$ & IM torque \\
\hline Method 6 & $\begin{array}{l}\text { MATLAB } \\
\text { Simscape }\end{array}$ & $\begin{array}{l}\text { Package } \\
\text { Solver }\end{array}$ & ode $15 \mathrm{~s}$ & $\begin{array}{l}\text { SM } q \text { axis } \\
\text { current }\end{array}$ \\
\hline
\end{tabular}
ode15s is a variable-order solver, ode 23 tb is based on an implicit Runge-Kutta strategy, and ode23t is a variation of the trapezoidal rule. Details are set forth in [5].

Table 3. Method Description
For each method, the variable with the largest error is used in the comparison and this signal differs between methods. Following a brief description of each method, a comparison study of the six methods is presented in Section VI.

\section{A. Method 1}

Method 1 utilizes MATLAB $\backslash$ Simscape and a variable-step, variable-order solver (ode15s). This solver offers low to medium accuracy and is implicit which is suitable for the stiffness of the system. It uses Numerical Differentiation Formulas (NDF) to generate the Jacobian matrices numerically [5]. In general, it is a multi-step solver that needs solutions of several preceding time-steps to compute the current state. The system differential algebraic equations (DAE) cause algebraic loops in MATLAB $\backslash$ Simulink, but MATLAB $\backslash$ Simscape is designed to solve DAEs therefore resolving the algebraic loop issue.

\section{B. Method 2}

The second method uses a dedicated solver to solve the algebraic loops in the system. This method begins by representing each component in the system by the following

$$
\left[\begin{array}{c}
v_{q} \\
v_{d}
\end{array}\right]=\left[\begin{array}{ll}
Z_{q q} & Z_{q d} \\
Z_{d q} & Z_{d d}
\end{array}\right]\left[\begin{array}{l}
i_{q} \\
i_{d}
\end{array}\right]+\left[\begin{array}{c}
E_{q} \\
E_{d}
\end{array}\right]
$$

or

$$
V=Z I+E
$$

Re-arranging this yields

$$
I=Y V-J
$$

where $Y$ is the inverse of $Z$ and $J$ is $Y E . \quad Y$ and $J$ are referred to as the admittance matrix and short circuit current vector respectively.

Solving the system of interconnection equations begins with observing two basic system relationships. The first is that the current into of the synchronous machine is the negative of the current in the cable, and the second is that the current in the cable is the sum of all the load currents. Sustituting (45) into the first relationship and manipulating yields

$$
\left(Y_{S M}-Y_{C B L}\right) V_{B 1}+Y_{L N} V_{B 2}=J_{S M}-J_{C B L}
$$

The same substitution in the second relationship results in

$$
Y_{C B L} V_{B 1}-\left(Y_{I M}+Y_{R L}+Y_{T R}\right) V_{B 2}=-J_{I M}-J_{R L}-J_{T R}
$$

Solving the system interconnection equations then translates to solving the following matrix multiplication obtained from (46) and (47). 


$$
\begin{aligned}
& {\left[\begin{array}{llll}
v_{q B 1} & v_{d B 1} & v_{q B 2} & v_{d B 2}
\end{array}\right]^{T}} \\
& =\left[\begin{array}{cc}
Y_{C B L} & -Y_{C B L}-Y_{I M}-Y_{R L}-Y_{T R} \\
Y_{S M}+Y_{C B L} & -Y_{C B L}
\end{array}\right]^{-1}\left[\begin{array}{c}
-J_{I M}-J_{R L}-J_{T R} \\
J_{S M}-J_{C B L}
\end{array}\right]
\end{aligned}
$$

Obtaining the admittance matrix and short circuit current vector for the system components is straightforward, except in the case of the transformer rectifier load. This is due to the non-linearity of this component.

The method for obtaining the admittance matrix and short circuit current vector of the transformer rectifier at each time step is shown in (49)-(53). First, the dc side voltage and current states are calculated using (34) and (35). Second, the $q$ and $d$ axis currents at the present operating point are obtained using

$$
\begin{gathered}
{\left[\begin{array}{l}
i_{q 0} \\
i_{d 0}
\end{array}\right]=f\left[\begin{array}{c}
v_{q 0} \\
v_{d 0}
\end{array}\right]} \\
{\left[\begin{array}{l}
i_{q 1} \\
i_{d 1}
\end{array}\right]=f\left[\begin{array}{c}
v_{q 0}+\Delta v_{q} \\
v_{d 0}
\end{array}\right]} \\
{\left[\begin{array}{l}
i_{q 2} \\
i_{d 2}
\end{array}\right]=f\left[\begin{array}{c}
v_{q 0} \\
v_{d 0}+\Delta v_{d}
\end{array}\right]}
\end{gathered}
$$

where $f[\cdot]$ refers to (36)- (41), and $v_{q 0}$ and $v_{d 0}$ are the $q$ and $d$ axis voltages of Bus 2. The load is numerically linearized around the present operating point using

$$
\left[\begin{array}{c}
i_{q} \\
i_{d}
\end{array}\right]=\left[\begin{array}{c}
i_{q 0} \\
i_{d 0}
\end{array}\right]+\underbrace{\left[\begin{array}{cc}
\frac{i_{q 1}-i_{q 0}}{\Delta v_{q}} & \frac{i_{q 2}-i_{q 0}}{\Delta v_{d}} \\
\frac{i_{d 1}-i_{d 0}}{\Delta v_{q}} & \frac{i_{d 2}-i_{d 0}}{\Delta v_{d}}
\end{array}\right]}_{Y_{T R}}\left[\begin{array}{c}
\Delta v_{q} \\
\Delta v_{d}
\end{array}\right]
$$

and

$$
J_{T R}=\left[\begin{array}{c}
i_{q 0} \\
i_{d 0}
\end{array}\right]-Y_{T R}\left[\begin{array}{c}
v_{q 0} \\
v_{d 0}
\end{array}\right]
$$

at each time step. The system of interconnection equations is solved using (48) to obtain the $q$ and $d$ axis voltages at each of the busses. This procedure iterates based upon the sum of the $\mathrm{q}$ and $\mathrm{d}$ axis voltage difference until an error threshold is met. This procedure effectively solves the non-linear algebraic loop using a Newton-Raphson approach.

\section{Method 3}

Analysis Method 3 uses the MATLAB \Simulink software package. Algebraic loops are resolved by introducing time delays using the Simulink "memory" block which delays the input by one integration time step. Instead of using the Simulink "discrete derivative" block, derivative terms were formulated using a backward difference approximation with second order error. In particular, in this approach the time derivative of state $x$ is approximated as

$$
\frac{d x}{d t} \approx \frac{3 x(t)-4 x(t-h)+x(t-2 h)}{2 h}
$$

where $h$ is the time step.

This is the principle difference between Method 3 and Method 4. The objective was to reduce simulation time compared to Method 4.

\section{Method 4}

Analysis method 4 uses the MATLAB $\backslash$ Simulink software package using discrete component models from the Simulink library and the MATLAB function blocks. At compilation time, MATLAB code in the function blocks is converted to C code which contributes to a faster solution. A Simulink discrete solver is used to advance the solution by a fixed time step $h=10^{-4} \mathrm{~s}$. Algebraic loops are resolved by introducing time delays equal to the time step $h$.

\section{E. Method 5}

Analysis method 5 also employs the MATLAB $\backslash$ Simulink software package with an implicit Simulink solver, ode23t. Algebraic loops are resolved by introducing time delays through the Simulink "memory" block which delays the input by one integration time step.

\section{F. Method 6}

Analysis method 6 uses the MATLAB $\backslash$ Simscape software package with ode15s. This method is similar to method 1 discussed earlier but programming approaches are slightly different. In analysis method 6 , the model is built using the Simscape language. The system equations described in section III are solved using a combination of custom equation based components, pre-built components, and functions present in the Simscape Foundation Library. Algebraic loops are handled internally by the solvers and do not appear as problems to be resolved, as is the case when they occur in Simulink models, in methods 3,4 , and 5 .

\section{SYSTEM SCENARIO}

In order to compare the different reduced-order modeling approaches considered herein, a time-domain simulation study is conducted. The system study scenario begins with all loads disconnected and the generator running at rated speed with no voltage at the terminals. The system is allowed to reach steady state $(t=5 s)$, whereupon the loads are connected simultaneously. In all of the figures depicting the system scenario, the solid line waveform is that of the left hand axis and the dashed line is that of the right hand axis. Fig. 6(a) depicts the transient response of the rms ac voltage and the speed of the synchronous machine. Fig. 6(b) depicts the response of the SM torque and power. The response of the SM 
rms current and field current are shown in Fig. 7(a) and the damper winding currents are shown in Fig. 7(b).
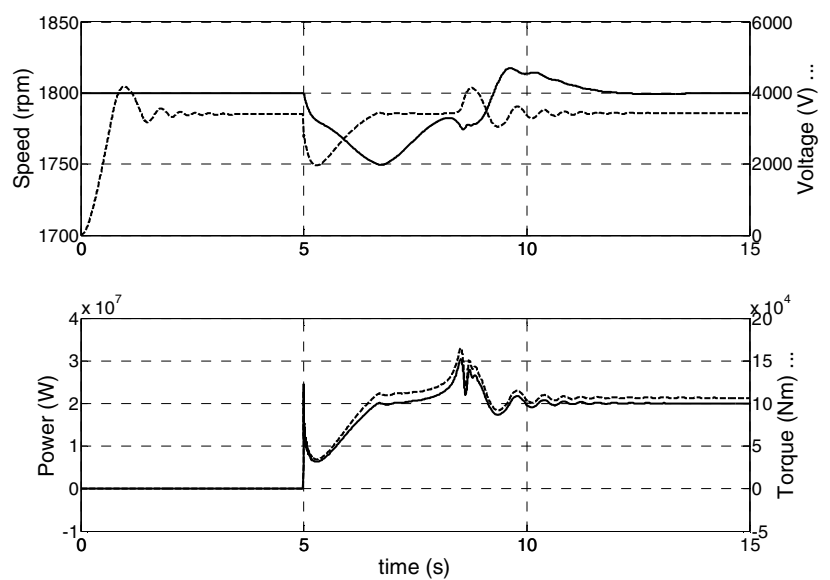

Figure 6. (a) SM Speed and Voltage (b) SM Power and Torque
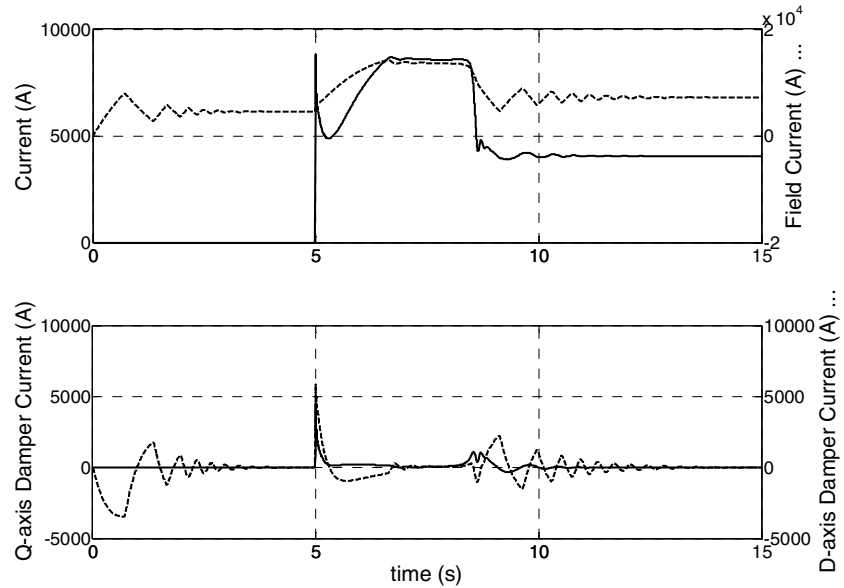

Figure 7. (a) SM RMS \& Field Currents (b) SM Damper Winding Currents
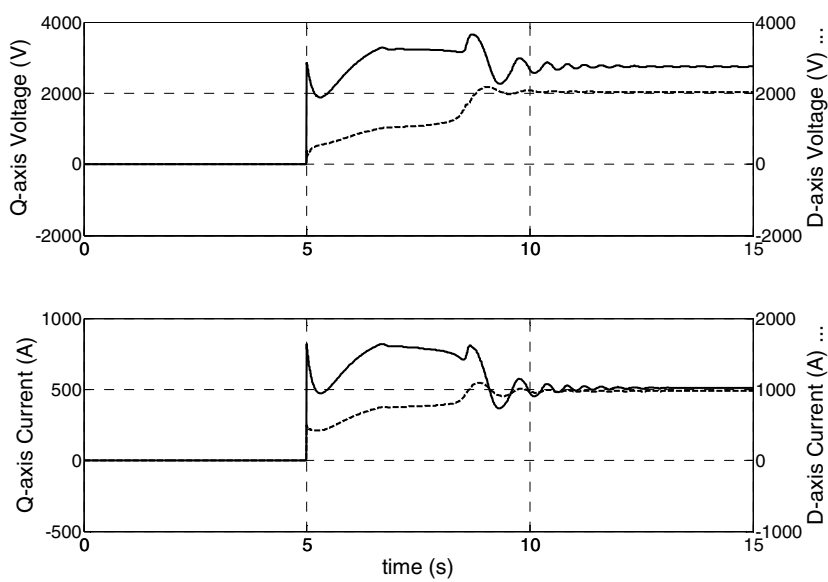

Figure 8 (a) CBL $q$ and $d$ axis Voltage (b) RL $q$ and $d$ axis Current
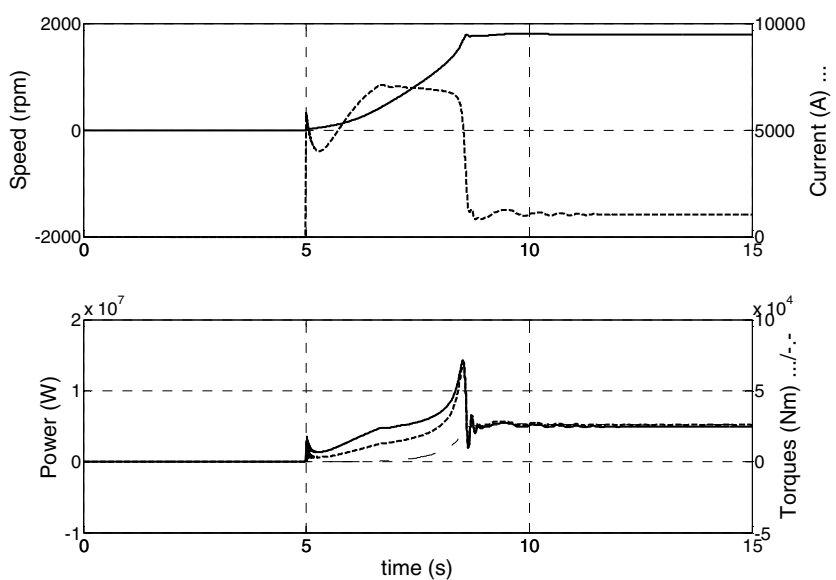

Figure 9. (a) IM Speed and Currents (b) IM Power and Torque
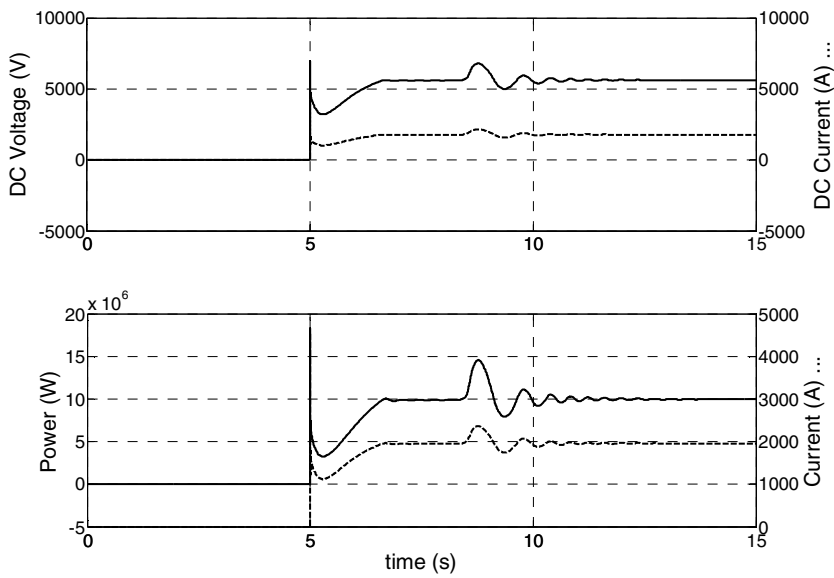

Figure 10. (a) TR DC voltage \& Current (b) TR Power \& Current

The waveforms of interest for the cable and RL load are depicted in Fig. 8. The $q$ and $d$ axis voltage of the cable and the $q$ and $d$ axis currents of the RL load are shown in Fig. 8(a) and Fig. 8(b) respectively.

The induction machine waveforms of interest are shown in Fig. 9. The speed and current of the induction machine are plotted in Fig. 9(a). Fig. 9(b) shows the power and torque of the induction machine. The electrical torque is represented with a dashed line and the load torque is represented with a dashed-dot line.

The waveforms of interest for the transformer rectifier load can be found in Fig. 10. Fig. 10(a) has the dc voltage and current of the transformer rectifier, and the power and rms current of the rectifier are shown Fig. 10(b).

It is relevant to note that all six modeling strategies yielded matching results.

\section{METHOD COMPARISON}

In order to compare the different modeling strategies effectively, an error metric is defined. It is a normalized mean square error which can be written as 


$$
e=\max _{i \in \text { selected signals }}
$$

where

$$
e_{i}=\sqrt{\frac{\int_{T_{1}}^{T_{2}}\left(x_{b, i}(t)-x_{i}(t)\right)^{2} d t}{\int_{T_{1}}^{T_{2}}\left(x_{b, i}(t)\right)^{2} d t}}
$$

where $x_{i}(t)$ is the signal of interest and $x_{b, i}(t)$ is the baseline data for that signal and model, which is taken to be that signal for the minimum tolerance value or time-step for that model.

The numerical error versus simulation time plots of all the modeling strategies are depicted together for comparison purposes in Fig. 11. The results are for a range of tolerance values in the variable-step algorithms and a range of time steps in the fixed-step algorithms. The horizontal red line is the selected maximum allowed error criteria for adequate accuracy. Two interesting claims can be withdrawn from the observation of the comparison plot in Fig. 11. First of all, it is observed that two clusters are formed. One group is for the methods 1, 2 and 6. And the other group is for the method 3, 4 and 5. With the first group being faster and more precise, it would appear that using numerical solution approach for the algebraic loop problem is more effective than the delay operator approach. Second, if one assumes that the user defined dedicated solver used in Method 2 is as effective as the package solver of Methods 1 and 6, it would appear that the Simscape based Method 1 and Method 6 outperformed the Simulink based Method 2. As a dedicated solver involves some effort, Methods 1 and 6 have an additional advantage over Method 2.

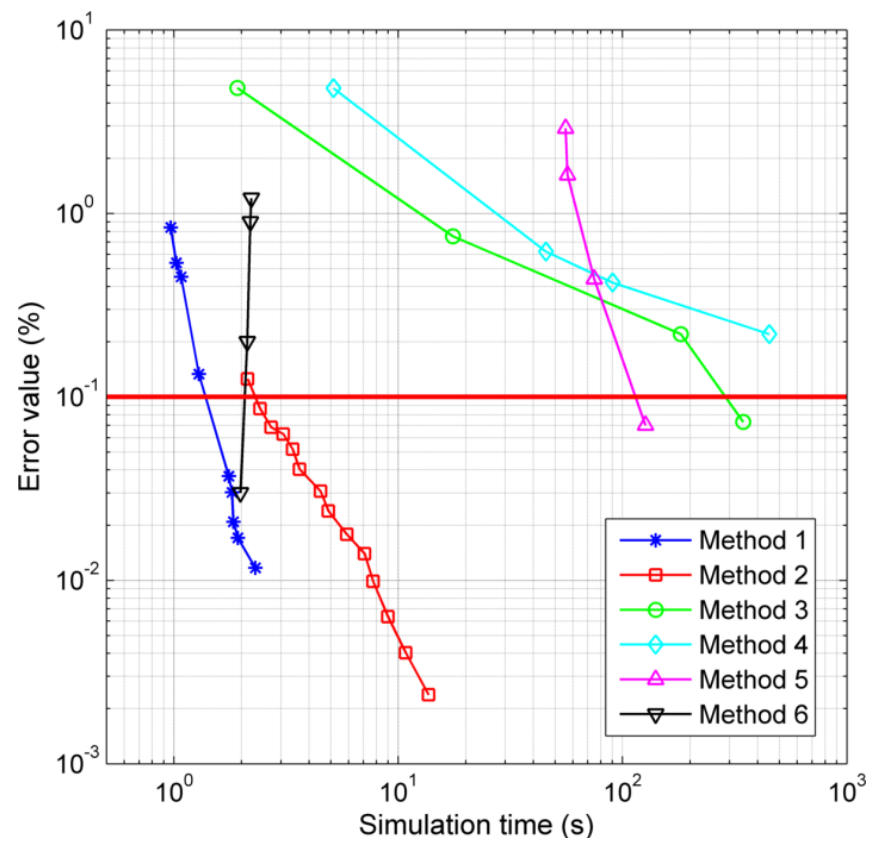

Figure 11. Method Comparison

\section{CONCLUSION}

A shipboard AC power system has been modeled in reduced-order average-value form using six different methods. A comparison of these methods in terms of error metric vs. simulation time has been presented. Based on this comparison, MATLAB $\backslash$ Simscape with its built in algebraic equation solver appeared to be the best choice for this type of simulation.

\section{REFERENCES}

[1] E. Zivi, M. Bash, R. Chan, J. Cider and C. Harianto, "Modeling, Simulation and Experimental Validation of a DC Power System Testbed," in Summer Computer Simulation Conference, Hague, Netherlands, 2011.

[2] J. Langstone et al., "Waveform-level Time-Domain Simulation Comparison Study of Three Shipboard Power System Architectures," in Summer Computer Simulation Conference 2012, Genoa, Italy, 2012.

[3] P. Krause, O. Wasynczuk and S. and Sudhoff, Analysis of Electric Machinery and Drive Systems, 2nd Edition, New York: John Wiley and Sons/IEEE Press,, 2002.

[4] J. Clerk Maxwell, A Treatise on Electricity and Magnetism, 3rd ed., vol. 2, Oxford: Clarendon, 1892, p. 68-73.

[5] The Mathworks, Inc., [Online]. Available: www.mathworks.com/help. 